\title{
Article \\ Brentuximab Vedotin and Pembrolizumab Combination in Patients with Relapsed/Refractory Hodgkin Lymphoma: A Single-Centre Retrospective Analysis
}

\author{
Fulvio Massaro ${ }^{1,2}{ }^{*}$, Nathalie Meuleman ${ }^{1}$, Dominique Bron ${ }^{1}$, Marie Vercruyssen ${ }^{1}$ (D) and Marie Maerevoet ${ }^{1}$ \\ 1 Department of Haematology, Institut Jules Bordet (ULB), 1070 Brussels, Belgium; \\ nathalie.meuleman@bordet.be (N.M.); dominique.bron@bordet.be (D.B.); \\ marie.vercruyssen@bordet.be (M.V.); marie.maerevoet@bordet.be (M.M.) \\ 2 PhD Program in Clinical and Experimental Medicine, University of Modena and Reggio Emilia, \\ 41121 Modena, Italy \\ * Correspondence: fulvio.massaro@bordet.be; Tel.: +32-025-417-214
}

check for updates

Citation: Massaro, F.; Meuleman, N.; Bron, D.; Vercruyssen, M.; Maerevoet, M. Brentuximab Vedotin and Pembrolizumab Combination in Patients with Relapsed/Refractory Hodgkin Lymphoma: A

Single-Centre Retrospective Analysis. Cancers 2022, 14, 982. https:// doi.org/10.3390/cancers14040982

Academic Editor: Daniel Molin

Received: 7 January 2022

Accepted: 3 February 2022

Published: 15 February 2022

Publisher's Note: MDPI stays neutral with regard to jurisdictional claims in published maps and institutional affiliations.

Copyright: (C) 2022 by the authors. Licensee MDPI, Basel, Switzerland. This article is an open access article distributed under the terms and conditions of the Creative Commons Attribution (CC BY) license (https:// creativecommons.org/licenses/by/ $4.0 /)$.
Simple Summary: The standard treatment for Hodgkin lymphoma (HL) patients presenting a relapsed/refractory $(\mathrm{R} / \mathrm{R})$ disease is salvage chemotherapy followed by autologous stem cell transplantation (ASCT). With commonly used chemotherapy combinations, 25-30\% fail to proceed to ASCT, with poor outcomes. The aim of this retrospective study was to evaluate the efficacy of brentuximab vedotin (BV) and pembrolizumab combination as a bridge to ASCT in R/R HL patients. We retrospectively collected data from 10 patients, 8 male and 2 female, with a median age of 30.7 years. The median follow-up time was 16.5 months, while the median number of received cycles of treatment was 4 (2-7). Eight patients proceeded to ASCT (80\%) and seven of them to subsequent $\mathrm{BV}$ maintenance, with two early disease progression (PD). The BV and pembrolizumab combination is a very effective bridge treatment to ASCT for high-risk R/R HL patients.

Abstract: Classical Hodgkin lymphoma (HL) patients presenting a relapsed/refractory $(\mathrm{R} / \mathrm{R})$ disease are currently managed with salvage chemotherapy followed by autologous stem cell transplantation (ASCT). However, almost $25-30 \%$ of these patients fail to achieve a complete response (CR) with standard salvage regimens. In this retrospective study, we evaluated the efficacy of a combination of brentuximab vedotin (BV) and pembrolizumab in a series of HL patients presenting with a high-risk, multi-refractory disease. Patients achieving a Deauville score $\leq 4$ proceeded to ASCT consolidation. After ASCT, patients received BV as maintenance for a total of 16 administrations. We collected data from 10 patients with a median age of 30.7 years. At a median follow-up of 16.5 months, we reported a complete metabolic remission (CMR) in eight patients (80\%), with seven patients (70\%) directly proceeding to ASCT (the other two patients in CMR are still undergoing treatment). BV consolidation was started in six patients and completed by three patients (one ongoing, two interruption). Two patients $(20 \%)$ presented a progressive disease (PD) and subsequently died, while the others are still in CMR. The BV and pembrolizumab combination is a very effective bridge treatment to ASCT for high-risk R/R HL patients.

Keywords: Hodgkin lymphoma; antibody-drug conjugate; immune checkpoint inhibition; brentuximab vedotin; pembrolizumab; salvage therapy; autologous stem cell transplantation

\section{Introduction}

Classical Hodgkin lymphoma (HL) is nowadays a highly curable disease, with standard first line polychemotherapy regimens achieving a complete remission (CR) rate of 80-90\%. However, $20-30 \%$ of patients will experience a relapse or a progressive disease (PD) [1-3]. The standard approach in this setting is salvage treatment followed by autologous stem cell transplantation (ASCT); almost half of the patients who undergo ASCT 
present long term disease remissions [4,5]. Several salvage chemotherapy schemes have been tested in this setting and mostly described in retrospective series; therefore, a gold standard treatment has not yet been identified. With commonly used chemotherapy combinations, $25-30 \%$ of these patients fail to achieve a complete metabolic response (CMR), which seems to be the most important prognostic factor to achieve a prolonged progressionfree survival (PFS) and to proceed to ASCT, with subsequently poor outcomes [6,7].

Single agents brentuximab vedotin (BV) and pembrolizumab have shown efficacy in heavily pretreated HL patients.

BV is an antibody-conjugated drug which exerts its cytotoxic action towards CD30positive HL cells. Its mechanism of action is based on the internalization of monomethyl auristatin E (MMAE), a microtubule-disrupting agent, into the target cell, inducing apoptosis as final result. In a phase 2 study conducted on R/R HL patients, BV induced an overall response rate (ORR) of $75 \%$ and a CR rate of $34 \%$ [8].

Pembrolizumab is a humanized monoclonal IgG4 kappa antibody directed against programmed death receptor-1 (PD-1) on lymphocytes. This receptor, which normally prevents the immune system from attacking itself, can be used by tumor cells to escape from anti-cancer immune response. Pembrolizumab monotherapy in R/R HL patients was associated with an ORR of $72 \%$ and a CR rate of $28 \%$ in the phase 2 KEYNOTE-087 trial [9].

The combination of BV with the PD-1 inhibitor nivolumab has been explored in a phase $1 / 2$ study as first salvage treatment for HL patients; this combination was associated with an ORR of $82 \%$ and a CR rate of $61 \%$ [10].

We performed a retrospective analysis of BV and pembrolizumab combination as salvage treatment in a series of heavily pretreated HL patients.

\section{Population and Methods}

We retrospectively collected data from ten consecutive HL patients presenting with a high-risk multi-refractory disease (two or more prior treatments), followed at Jules Bordet Institute between May 2019 and October 2021 and treated with a combination of BV and pembrolizumab.

All enrolled patients had biopsy-proven R/R HL and had an ${ }^{18}$ FDG-PET-CT (PET-CT) avid measurable disease. Patients were covered by special insurance conditions permitting treatment administration and reimbursement. Treatment proposal was approved by our multidisciplinary oncology committee and informed consent was obtained from all subjects receiving the salvage combination.

Treatment consisted of a combination of BV $(1.8 \mathrm{mg} / \mathrm{kg}$ IV) and pembrolizumab (200 mg IV fixed dose), delivered in 3-week cycles. A PET-CT evaluation was performed after two cycles: patients achieving a Deauville score (DS) $\leq 4$ received high-dose chemotherapy (carmustine, etoposide, cytarabine, melphalan) and ASCT consolidation. Responses were assessed according to the Revised Response Criteria for Malignant Lymphoma [11,12]. Patients could continue to receive further cycles of BV and pembrolizumab before ASCT, at the physician's discretion. After ASCT, patients received BV as maintenance for a total of 16 administrations (including pre-ASCT cycles). A PET-CT evaluation was performed 90 days after ASCT and at the end of maintenance treatment.

Toxicity was reported according to Common Terminology Criteria for Adverse Events (CTCAE) score v.5.0.

\section{Results}

We retrospectively analyzed data from 10 patients, 8 males and 2 females, with a median age of 30.7 (20.6-36.4) years. Patients had received a median of 3 (2-5) prior lines of treatment, and the median time from diagnosis to treatment with pembrolizumab and BV combination was 27.7 months.

Among baseline characteristics, nine (90\%) patients presented an advanced disease at relapse, six $(60 \%)$ a primary refractory disease, six $(60 \%)$ extranodal disease at relapse and four $(40 \%)$ a CR duration less than 12 months. Five (50\%) patients presented three of these 
features simultaneously. Table 1 summarizes demographic and baseline characteristics for all enrolled patients.

Table 1. Baseline characteristics of patients $(n=10)$.

\begin{tabular}{ccc}
\hline \multicolumn{1}{c}{ Characteristics } & Patients, $\boldsymbol{n}$. \\
\hline Median age, years (range) & & $30.7(20.6-36.4)$ \\
\hline Sex, male/female & & $8(80 \%) / 2(20 \%)$ \\
\hline $\begin{array}{c}\text { Median time from diagnosis to } \\
\text { pembro-BV, months (range) }\end{array}$ & $27.7(13.6-51)$ \\
\hline First-line treatment & ABVD & $5(50 \%)$ \\
& BEACOPP esc & $4(40 \%)$ \\
Number of prior treatments & CHOEP & $1(10 \%)$ \\
before & 2 & $3(30 \%)$ \\
pembro-BV salvage & 3 & $6(60 \%)$ \\
First salvage therapy & 5 & $1(10 \%)$ \\
\hline Second salvage therapy & DHAP & $6(60 \%)$ \\
& BEGEV & $3(30 \%)$ \\
& ICE & $1(10 \%)$ \\
\hline Refractory disease & BEGEV & $2(20 \%)$ \\
\hline Complete remission $<12$ months & Bendamustine & $2(20 \%)$ \\
\hline Extranodal involvement at relapse & ICE & $1(10 \%)$ \\
\hline Advanced stage at relapse & BEACOPP esc & $1(10 \%)$ \\
\hline ABVD, & & $6(60 \%)$ \\
\hline
\end{tabular}

ABVD, doxorubicin, bleomycin, vinblastine, dacarbazine; BEACOPP esc, doxorubicin, cyclophosphamide, etoposide, procarbazine, prednisolone, bleomycin, vincristine; CHOEP, cyclophosphamide, doxorubicin, vincristine, etoposide, prednisolone; BEGEV, bendamustine, gemcitabine, vinorelbine; DHAP, dexamethasone, cytarabine, cisplatin; ICE, ifosfamide, carboplatin, etoposide; BV, brentuximab vedotin.

All patients completed the salvage treatment and had a PET-CT evaluation. The median follow-up time was 16.5 (2.4-29.9) months, while the median number of cycles was $4(2-7)$.

The ORR was 90\%, and particularly, a CMR was achieved from eight patients (80\%), with a median time to best response of two cycles. Among the group of responding patients, seven (70\%) proceeded to ASCT: in one case, a patient in CMR (DS1) after two cycles presented a DS4 after six cycles (salvage treatment pursued due to ASCT deferral because of the first wave of COVID-19 pandemic) and received consolidative radiotherapy before ASCT. Two patients are scheduled to receive ASCT shortly.

Stem cell collection was successful for seven out of seven patients, with a median of $5 \times 10^{6} \mathrm{CD} 34+$ cells $/ \mathrm{kg}$ collected. Mobilizing agents included granulocyte colonystimulating factor (G-CSF) in four cases and G-CSF with plerixafor in three cases. Concerning the other patients, two are expected to be collected in the next weeks while another one was collected before starting the salvage treatment.

The only patient presenting a PD after two cycles was treated with a combination of nivolumab and gemcitabine, achieving a partial response (PR) and subsequently received radiotherapy and ASCT followed by allogeneic stem cell transplantation (alloSCT) in a tandem strategy.

BV consolidation was started at a median of 36 (28-85) days after ASCT from six patients and completed by three of them. One patient is undergoing treatment while two others interrupted consolidation (one PD, one consolidation with alloSCT). 
Two patients (20\%) presented with PD after ASCT: one patient was in CMR and the other in PR (with a DS5) before ASCT and they relapsed 4 months and 3 months after the procedure, respectively. One patient was treated with a combination of nivolumab and gemcitabine and achieved a CR, while the other was treated with a combination of nivolumab and bendamustine and achieved a PR. Both patients received a consolidation with alloSCT and died from infectious complications (systemic tuberculosis and COVID-19 infection in one case, septic shock in one case). Median progression-free survival (PFS) value was 12.9 months. Table 2 describes in detail the treatment and the outcome of each patient. Figure 1 shows the overall survival (OS) curve.

Table 2. Treatment details for each patient in study.

\begin{tabular}{|c|c|c|c|c|c|c|c|c|c|c|}
\hline & $\begin{array}{c}\text { Prior Lines of } \\
\text { Treatment }\end{array}$ & $\begin{array}{c}\text { Pembro + } \\
\text { BV } \\
\text { (Cycles) }\end{array}$ & PET2 & ASCT & $\begin{array}{c}\text { BV } \\
\text { Post-ASCT } \\
\text { (Cycles) }\end{array}$ & $\begin{array}{l}\text { PD after } \\
\text { ASCT }\end{array}$ & $\begin{array}{l}\text { Allo } \\
\text { SCT }\end{array}$ & $\begin{array}{c}\text { Follow- } \\
\text { Up } \\
\text { (Months) }\end{array}$ & $\begin{array}{c}\text { Last } \\
\text { Disease } \\
\text { Status }\end{array}$ & $\begin{array}{c}\text { Patient } \\
\text { Status }\end{array}$ \\
\hline Pt1 & 3 & 2 & DS3 & Yes & 14 & No & No & 29.9 & CR & Alive \\
\hline Pt2 & 2 & 4 & DS3 & Yes & 5 & Yes & Yes a & 17.4 & CR & Dead \\
\hline $\mathrm{Pt} 3$ & 3 & 7 & DS4 & Yes & 9 & No & No & 27.1 & $\mathrm{CR}$ & Alive \\
\hline Pt4 & 3 & 2 & DS2 & Yes & 14 & No & No & 27.6 & $\mathrm{CR}$ & Alive \\
\hline $\mathrm{Pt} 5$ & 2 & 4 & DS5 & No b & No & Yes & Yes & 10.1 & PR & Dead \\
\hline Pt6 & 2 & 6 & DS1 & Yes (+RT) & 3 & No & Yes c & 22.4 & $\mathrm{CR}$ & Alive \\
\hline Pt7 & 3 & 6 & DS2 & Yes & 1 & No & No & 21.7 & $\mathrm{CR}$ & Alive \\
\hline $\mathrm{Pt} 8$ & 3 & 4 & DS1 & Yes & Not yet & NA & No & 3.3 & $\mathrm{CR}$ & Alive \\
\hline Pt9 & 5 & 4 & DS1 & Not yet & Not yet & NA & No & 2.4 & $\mathrm{CR}$ & Alive \\
\hline Pt10 & 3 & 4 & DS2 & Not yet & Not yet & NA & No & 2.6 & $\mathrm{CR}$ & Alive \\
\hline
\end{tabular}

a After subsequent treatment line (nivolumab + gemcitabine) due to PD after ASCT. ${ }^{\mathrm{b}}$ ASCT after subsequent treatment line (nivolumab + gemcitabine and RT) due to PD. ${ }^{c}$ Directly after ASCT (tandem strategy).

Overall Survival

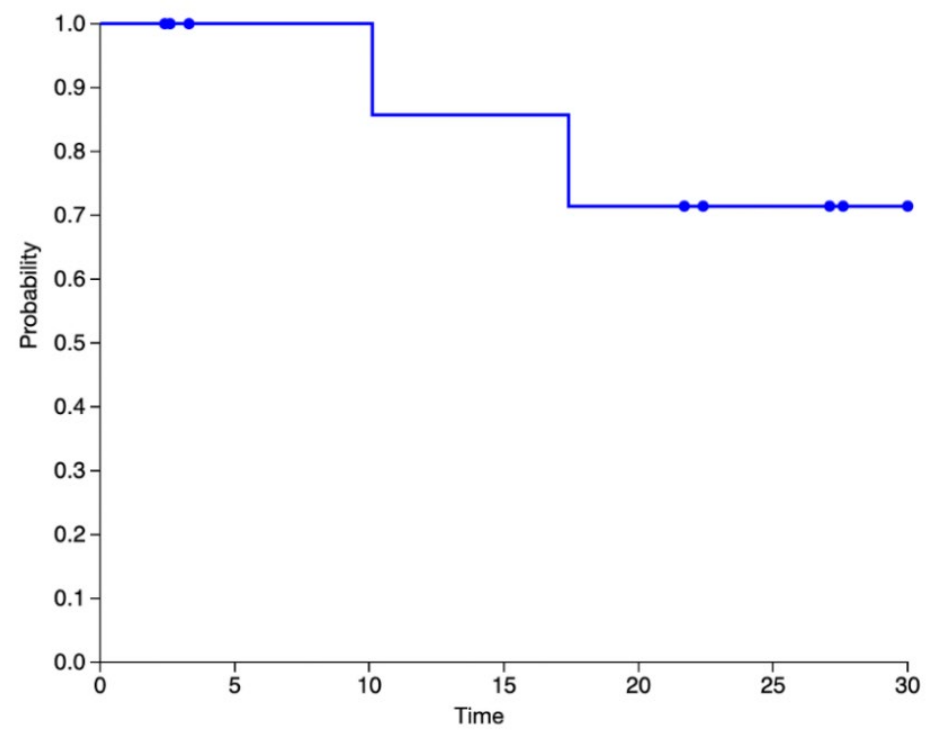

Figure 1. Kaplan-Meier curve for OS.

Three patients (30\%) experienced four adverse events (AEs) during the treatment with pembrolizumab and BV: one case of infusion-related reaction, neutropenia, polyarthritis and hyperthyroidism, respectively, all classified as grade 3 events. Polyarthritis was managed with pembrolizumab discontinuation and systemic corticosteroids treatment, hyperthyroidism with pembrolizumab discontinuation only. Two patients (33\%) presented 
two AEs during BV consolidation: a grade 2 arthralgia and a grade 4 neutropenia. Both cases were successfully managed with BV dose reduction to $1.2 \mathrm{mg} / \mathrm{kg}$.

\section{Discussion}

To the best of our knowledge, no data are currently available concerning the combination of BV and pembrolizumab in R/R HL. We described the outcomes of 10 patients treated with this combination, reporting a CMR of $80 \%$ and a total of $70 \%$ of patients proceeding to ASCT directly after this line of treatment. Moreover, response achievement was commonly quick, with most patients in CMR after only two cycles. The safety profile was manageable, with only a few grade 3 events to be mentioned. It has to be underlined that treatment schedule is compatible with outpatient administration.

Herrera and colleagues explored the efficacy of the combination of BV with another PD1 inhibitor, nivolumab; a recently published update reported an ORR of $85 \%$ and a CR rate of $67 \%$, with a 3-year PFS of $77 \%$ for the entire cohort and $91 \%$ for patients undergoing ASCT directly after study treatment. The safety profile was manageable, mainly characterized by grade $1-2$ events and $18 \%$ of immune related adverse events (IrAE) needing corticosteroids treatment. It is difficult to perform a true comparison among these studies; however, it is interesting to note that in the trial with nivolumab patients were less pretreated (no prior salvage treatments received) and presented less frequently unfavorable disease characteristics ( $45 \%$ of primary refractory, 31\% of CR duration less than 12 months, $26 \%$ of extranodal disease at relapse) when compared to our series [10,13].

The synergistic action of BV with a PD-1 inhibitor seems to rely on the combination of tumor microenvironment modulation and T-cell clonal expansion. Interestingly, if the inhibitory activity towards regulatory $\mathrm{T}$ cells has already been described after single-agent $\mathrm{BV}$, the promotion of T-cell clonal expansion after single-agent PD-1 inhibitor has not yet been observed [10].

Hence, this combination could lead to a significant improvement for those heavily pretreated patients considered as chemorefractory. In restoring chemosensitivity, some of these patients could be spared from alloSCT, which is burdened with an important treatment-related mortality.

It has been reported in several series that even patients who failed PD1 inhibitor therapy seem to benefit from a re-treatment with chemotherapy, supporting the hypothesis that these drugs can re-sensitize lymphoma cells to conventional treatment after previous failure [14,15]. In a series of $30 \mathrm{R} / \mathrm{R}$ HL patients from the LYSA (Lymphoma Study Association) group treated with chemotherapy after unsatisfactory response on PD1 inhibitor treatment, the reported ORR was $67 \%$ for the proportion of patients presenting a $\mathrm{PD}$, with a CR rate of $46 \%$. Several patients achieved a response despite being re-exposed to the same chemotherapy agent received before PD1 inhibitor treatment. Furthermore, patients treated with a combination of PD1 inhibitors and chemotherapy showed a trend to a better response than those treated only with chemotherapy, underlining the potential synergy among the two treatments [16]. Similarly, in a retrospective analysis, $81 \mathrm{R} / \mathrm{R} \mathrm{HL}$ patients from the United States and Canada were treated with several types of salvage chemotherapy after PD1 inhibitors failure: the ORR was $62 \%$, the CR rate of $42 \%$ and $47 \%$ of patients could proceed to ASCT and/or alloSCT. Interestingly, patients presenting a PD after anti-PD1 treatment were also capable of achieving a response after being treated with subsequent conventional chemotherapy [17].

The potential role of PD-1 inhibitors as chemosensitizing agents has also been studied in the setting of pre-ASCT R/R HL. In a retrospective analysis, heavily pre-treated high-risk HL patients (median of three prior systemic lines of treatment, $62 \%$ of primary refractory disease) received a PD-1 inhibitor, as a single agent or in combination with chemotherapy, before ASCT; at a median follow-up of 20 months, the PFS for the entire cohort was $81 \%$ with no significant differences among patients presenting a positive or a negative PET-CT (18-month PFS, $75 \%$ vs. $85 \%, p=0.18$ ) [18]. However, it must be underlined that this study did not include a centralized radiologic review and that PET-CT was not assessed using 
response criteria explicitly designed for immunotherapy in lymphoma, such as LYRIC or RECIL [12,19].

The early introduction of PD1 inhibitors for HL salvage treatment has been explored by Moskowitz and colleagues in a recently published study using a combination of pembrolizumab and GVD (gemcitabine, vinorelbine and liposomal doxorubicin) chemotherapy as first salvage treatment followed by ASCT. This association showed an impressive CMR rate of $95 \%$ with no PD reported after 1 year of follow-up [20]. Authors hypothesize that one or more drugs within GVD are capable of enhancing the anti-tumoral activity of pembrolizumab, according to preclinical data which showed that both gemcitabine and doxorubicin present a stimulating activity towards T cell-mediated immunity [21,22].

The combination of BV with chemotherapy (bendamustine, GVD, IGEV, ESHAP, DHAP) has also been explored, with encouraging results (CR rates $70-90 \%$ ); however, it must be underlined that these schemes are markedly more toxic (G3-4 hematological toxicity $50-90 \%$ ) and seem to be less effective in primary refractory patients [23-30].

Our analysis presents limitations due to its retrospective nature, its small size and the absence of biological studies. However, it supports the idea of combining BV and Pd1 inhibitors in relapsed/refractory HL patients. Particularly, we think that this combination is an extremely valid option for HL patients' refractory to chemotherapy-based salvage treatment.

\section{Conclusions}

Our preliminary data suggest that the BV and pembrolizumab combination is a highly effective and safe bridge treatment to ASCT for high-risk, heavily pretreated R/R HL patients. Compared to other salvage regimens, the efficacy is also confirmed in primary refractory patients. Further studies on larger samples are needed to confirm these data.

Author Contributions: F.M. followed patients during treatment, collected and analyzed data, and wrote the manuscript; M.V. and M.M. followed patients during treatment and revised the manuscript; D.B. and N.M. revised the manuscript. All authors have read and agreed to the published version of the manuscript.

Funding: This research received no external funding.

Institutional Review Board Statement: The study was conducted in accordance with the Declaration of Helsinki and approved by the Ethics Committee of Institut Jules Bordet (CE3440).

Informed Consent Statement: Informed consent was obtained from all subjects involved in the study.

Data Availability Statement: The data presented in this study are available on request from the corresponding author.

Conflicts of Interest: The authors declare no conflict of interest.

\section{References}

1. André, M.P.E.; Girinsky, T.; Federico, M.; Reman, O.; Fortpied, C.; Gotti, M.; Casasnovas, O.; Brice, P.; van der Maazen, R.; Re, A.; et al. Early Positron Emission Tomography Response-Adapted Treatment in Stage I and II Hodgkin Lymphoma: Final Results of the Randomized EORTC/LYSA/FIL H10 Trial. J. Clin. Oncol. 2017, 35, 1786-1794. [CrossRef] [PubMed]

2. Barrington, S.F.; Kirkwood, A.A.; Franceschetto, A.; Fulham, M.J.; Roberts, T.H.; Almquist, H.; Brun, E.; Hjorthaug, K.; Viney, Z.N.; Pike, L.C.; et al. PET-CT for Staging and Early Response: Results from the Response-Adapted Therapy in Advanced Hodgkin Lymphoma Study. Blood 2016, 127, 1531-1538. [CrossRef] [PubMed]

3. Press, O.W.; Li, H.; Schöder, H.; Straus, D.J.; Moskowitz, C.H.; LeBlanc, M.; Rimsza, L.M.; Bartlett, N.L.; Evens, A.M.; Mittra, E.S.; et al. US Intergroup Trial of Response-Adapted Therapy for Stage III to IV Hodgkin Lymphoma Using Early Interim Fluorodeoxyglucose-Positron Emission Tomography Imaging: Southwest Oncology Group S0816. J. Clin. Oncol. 2016, 34, 2020-2027. [CrossRef]

4. Linch, D.C.; Winfield, D.; Goldstone, A.H.; Moir, D.; Hancock, B.; McMillan, A.; Chopra, R.; Milligan, D.; Hudson, G.V. Dose Intensification with Autologous Bone-Marrow Transplantation in Relapsed and Resistant Hodgkin's Disease: Results of a BNLI Randomised Trial. Lancet 1993, 341, 1051-1054. [CrossRef] 
5. Schmitz, N.; Pfistner, B.; Sextro, M.; Sieber, M.; Carella, A.M.; Haenel, M.; Boissevain, F.; Zschaber, R.; Müller, P.; Kirchner, H.; et al. Aggressive Conventional Chemotherapy Compared with High-Dose Chemotherapy with Autologous Haemopoietic Stem-Cell Transplantation for Relapsed Chemosensitive Hodgkin's Disease: A Randomised Trial. Lancet 2002, 359, 2065-2071. [CrossRef]

6. Moskowitz, A.J.; Schöder, H.; Gavane, S.; Thoren, K.L.; Fleisher, M.; Yahalom, J.; McCall, S.J.; Cadzin, B.R.; Fox, S.Y.; Gerecitano, J.; et al. Prognostic Significance of Baseline Metabolic Tumor Volume in Relapsed and Refractory Hodgkin Lymphoma. Blood 2017, 130, 2196-2203. [CrossRef] [PubMed]

7. LaCasce, A.S. Treating Hodgkin Lymphoma in the New Millennium: Relapsed and Refractory Disease. Hematol. Oncol. 2019, 37, 87-91. [CrossRef]

8. Younes, A.; Gopal, A.K.; Smith, S.E.; Ansell, S.M.; Rosenblatt, J.D.; Savage, K.J.; Ramchandren, R.; Bartlett, N.L.; Cheson, B.D.; de Vos, S.; et al. Results of a Pivotal Phase II Study of Brentuximab Vedotin for Patients with Relapsed or Refractory Hodgkin's Lymphoma. JCO 2012, 30, 2183-2189. [CrossRef]

9. Chen, R.; Zinzani, P.L.; Lee, H.J.; Armand, P.; Johnson, N.A.; Brice, P.; Radford, J.; Ribrag, V.; Molin, D.; Vassilakopoulos, T.P.; et al Pembrolizumab in Relapsed or Refractory Hodgkin Lymphoma: 2-Year Follow-up of KEYNOTE-087. Blood 2019, 134, 1144-1153. [CrossRef]

10. Herrera, A.F.; Moskowitz, A.J.; Bartlett, N.L.; Vose, J.M.; Ramchandren, R.; Feldman, T.A.; LaCasce, A.S.; Ansell, S.M.; Moskowitz C.H.; Fenton, K.; et al. Interim Results of Brentuximab Vedotin in Combination with Nivolumab in Patients with Relapsed or Refractory Hodgkin Lymphoma. Blood 2018, 131, 1183-1194. [CrossRef]

11. Cheson, B.D.; Fisher, R.I.; Barrington, S.F.; Cavalli, F.; Schwartz, L.H.; Zucca, E.; Lister, T.A. Recommendations for Initial Evaluation, Staging, and Response Assessment of Hodgkin and Non-Hodgkin Lymphoma: The Lugano Classification. J. Clin. Oncol. 2014, 32, 3059-3068. [CrossRef]

12. Cheson, B.D.; Ansell, S.; Schwartz, L.; Gordon, L.I.; Advani, R.; Jacene, H.A.; Hoos, A.; Barrington, S.F.; Armand, P. Refinement of the Lugano Classification Lymphoma Response Criteria in the Era of Immunomodulatory Therapy. Blood 2016, 128, 2489-2496. [CrossRef]

13. Advani, R.H.; Moskowitz, A.J.; Bartlett, N.L.; Vose, J.M.; Ramchandren, R.; Feldman, T.A.; LaCasce, A.S.; Christian, B.A.; Ansell, S.M.; Moskowitz, C.H.; et al. Brentuximab Vedotin in Combination with Nivolumab in Relapsed or Refractory Hodgkin Lymphoma: 3-Year Study Results. Blood 2021, 138, 427-438. [CrossRef]

14. Casadei, B.; Argnani, L.; Morigi, A.; Lolli, G.; Broccoli, A.; Pellegrini, C.; Nanni, L.; Stefoni, V.; Coppola, P.E.; Carella, M.; et al. Effectiveness of Chemotherapy after Anti-PD-1 Blockade Failure for Relapsed and Refractory Hodgkin Lymphoma. Cancer Med. 2020, 9, 7830-7836. [CrossRef]

15. Casadei, B.; Argnani, L.; Morigi, A.; Lolli, G.; Broccoli, A.; Pellegrini, C.; Nanni, L.; Stefoni, V.; Coppola, P.E.; Carella, M.; et al. Potential Survival Benefit for Patients Receiving Autologous Hematopoietic Stem Cell Transplantation after Checkpoint Inhibitors for Relapsed/Refractory Hodgkin Lymphoma: A Real-Life Experience. Hematol. Oncol. 2020, 38, 737-741. [CrossRef]

16. Rossi, C.; Gilhodes, J.; Maerevoet, M.; Herbaux, C.; Morschhauser, F.; Brice, P.; Garciaz, S.; Borel, C.; Ysebaert, L.; Obéric, L.; et al. Efficacy of Chemotherapy or Chemo-Anti-PD-1 Combination after Failed Anti-PD-1 Therapy for Relapsed and Refractory Hodgkin Lymphoma: A Series from Lysa Centers. Am. J. Hematol. 2018, 93, 1042-1049. [CrossRef]

17. Carreau, N.A.; Pail, O.; Armand, P.; Merryman, R.; Advani, R.H.; Spinner, M.A.; Herrera, A.; Chen, R.; Tomassetti, S.; Ramchandren, R.; et al. Checkpoint Blockade Treatment May Sensitize Hodgkin Lymphoma to Subsequent Therapy. Oncologist 2020, 25, 878-885. [CrossRef]

18. Merryman, R.W.; Redd, R.A.; Nishihori, T.; Chavez, J.; Nieto, Y.; Darrah, J.M.; Rao, U.; Byrne, M.T.; Bond, D.A.; Maddocks, K.J.; et al. Autologous Stem Cell Transplantation after Anti-PD-1 Therapy for Multiply Relapsed or Refractory Hodgkin Lymphoma. Blood Adv. 2021, 5, 1648-1659. [CrossRef]

19. Younes, A.; Hilden, P.; Coiffier, B.; Hagenbeek, A.; Salles, G.; Wilson, W.; Seymour, J.F.; Kelly, K.; Gribben, J.; Pfreunschuh, M.; et al. International Working Group Consensus Response Evaluation Criteria in Lymphoma (RECIL 2017). Ann. Oncol. 2017, 28, 1436-1447. [CrossRef]

20. Moskowitz, A.J.; Shah, G.; Schöder, H.; Ganesan, N.; Drill, E.; Hancock, H.; Davey, T.; Perez, L.; Ryu, S.; Sohail, S.; et al. Phase II Trial of Pembrolizumab Plus Gemcitabine, Vinorelbine, and Liposomal Doxorubicin as Second-Line Therapy for Relapsed or Refractory Classical Hodgkin Lymphoma. J. Clin. Oncol. 2021. [CrossRef]

21. Duffy, A.G.; Greten, T.F. Immunological Off-Target Effects of Standard Treatments in Gastrointestinal Cancers. Ann. Oncol. 2014, 25, 24-32. [CrossRef]

22. Galluzzi, L.; Senovilla, L.; Zitvogel, L.; Kroemer, G. The Secret Ally: Immunostimulation by Anticancer Drugs. Nat. Rev. Drug Discov. 2012, 11, 215-233. [CrossRef]

23. Driessen, J.; Tonino, S.H.; Moskowitz, A.J.; Kersten, M.J. How to Choose First Salvage Therapy in Hodgkin Lymphoma: Traditional Chemotherapy vs Novel Agents. Hematology 2021, 2021, 240-246. [CrossRef]

24. LaCasce, A.S.; Bociek, G.; Sawas, A.; Caimi, P.F.; Agura, E.; Matous, J.; Ansell, S.; Crosswell, H.; Islas-Ohlmayer, M.; Behler, C.; et al. Brentuximab Vedotin Plus Bendamustine: A Highly Active Salvage Treatment Regimen for Patients with Relapsed or Refractory Hodgkin Lymphoma. Blood 2015, 126, 3982. [CrossRef]

25. Broccoli, A.; Argnani, L.; Botto, B.; Corradini, P.; Pinto, A.; Re, A.; Vitolo, U.; Fanti, S.; Stefoni, V.; Zinzani, P.L. First Salvage Treatment with Bendamustine and Brentuximab Vedotin in Hodgkin Lymphoma: A Phase 2 Study of the Fondazione Italiana Linfomi. Blood Cancer J. 2019, 9, 100. [CrossRef] 
26. Bartlett, N.L.; Niedzwiecki, D.; Johnson, J.L.; Friedberg, J.W.; Johnson, K.B.; van Besien, K.; Zelenetz, A.D.; Cheson, B.D.; Canellos, G.P. Cancer Leukemia Group B Gemcitabine, Vinorelbine, and Pegylated Liposomal Doxorubicin (GVD), a Salvage Regimen in Relapsed Hodgkin's Lymphoma: CALGB 59804. Ann. Oncol. 2007, 18, 1071-1079. [CrossRef] [PubMed]

27. Abuelgasim, K.A.; Alzahrani, M.; Alsharhan, Y.; Khairi, M.; Hommady, M.; Gmati, G.; Salama, H.; Ali, O.; Alahmari, B.; Masuadi, E.M.; et al. Chemoimmunotherapy with Brentuximab Vedotin Combined with Ifosfamide, Gemcitabine, and Vinorelbine Is Highly Active in Relapsed or Refractory Classical Hodgkin Lymphoma. Bone Marrow Transplant. 2019, 54, 1168-1172. [CrossRef]

28. Garcia-Sanz, R.; Sureda, A.; Gonzalez, A.P.; De la Cruz, F.; Sanchez-Gonzalez, B.; Rodriguez, A.; Domingo-Domenech, E.; Miriam, M.; Miriam, M.; Lopez, J.; et al. Brentuximab Vedotin Plus ESHAP (BRESHAP) Is a Highly Effective Combination for Inducing Remission in Refractory and Relapsed Hodgkin Lymphoma Patients Prior to Autologous Stem Cell Transplant: A Trial of the Spanish Group of Lymphoma and Bone Marrow Transplantation (GELTAMO). Blood 2016, 128, 1109. [CrossRef]

29. Kersten, M.J.; Driessen, J.; Zijlstra, J.M.; Plattel, W.J.; Morschhauser, F.; Lugtenburg, P.J.; Brice, P.; Hutchings, M.; Gastinne, T.; Liu, R.; et al. Combining Brentuximab Vedotin with Dexamethasone, High-Dose Cytarabine and Cisplatin as Salvage Treatment in Relapsed or Refractory Hodgkin Lymphoma: The Phase II HOVON/LLPC Transplant BRaVE Study. Haematologica 2021, 106, 1129-1137. [CrossRef]

30. Moskowitz, A.J.; Herrera, A.F.; Beaven, A.W. Relapsed and Refractory Classical Hodgkin Lymphoma: Keeping Pace with Novel Agents and New Options for Salvage Therapy. Am. Soc. Clin. Oncol. Educ. Book 2019, 39, 477-486. [CrossRef] 